\title{
ALGEBRAIC EXTENSIONS OF CONTINUOUS FUNCTION ALGEBRAS
}

\author{
G. A. HEUER AND J. A. LINDBERG, JR. ${ }^{1}$
}

1. Introduction. In [1] it is shown that the algebraic extension $B=A[x] /(\alpha(x))$, where $A$ is a commutative Banach algebra (with unit) and $\alpha(x) \in A[x]$ is a monic polynomial, can be normed so that it is a Banach algebra and the canonical mapping of $A$ into $B$ is an isometry as well as an isomorphism. It can be shown that if $A$ is semi-simple, then $B$ is semi-simple if and only if the discriminant $d$ of $\alpha(x)$ is not a zero divisor in $A$ (cf. [1] and [2]).

In the present note, we consider only algebras $A$ of continuous functions which are complete in the sup norm, and study the relationship of the sup norm completeness (hereafter, just "completeness") of the Gelfand representation $B^{\wedge}$ of $B$ to certain properties of the polynomial $\alpha(x)$. Our main theorem may be regarded as the topological analog of the above result about the semi-simplicity of $B$. It states that if $B$ is semi-simple, (i.e., $d$ is not a zero divisor in $A$ ), then $B^{\wedge}$ is sup norm complete if and only if $d$ is not a topological zero divisor in $A$. In $\$ 3$, we introduce the concept of singular point of a monic polynomial and show that if $\alpha(x)$ has no singular points, then $B^{\wedge}$ is complete. An example (1) shows that $B^{\wedge}$ may be complete even if $B$ is not semi-simple ( $d$ is a zero divisor in $A$ ) and $\alpha(x)$ has singular points. Special attention is paid to the case where $A=C(\Omega), \Omega$ a compact Hausdorff space.

2. The main theorem. Before going on to the theorem, we make the following comments. The sup norm of a function $f$ is to be denoted by $|f|$.

Let $\Omega$ be a compact Hausdorff space and let $A \subseteq C(\Omega)$ be a closed subalgebra which separates the points of $\Omega$ and contains the constant functions on $\Omega$. We assume that the carrier space [5] of $A$ is $\Omega$, that is, each multiplicative linear functional on $A$ to $C$ (the complex numbers) is given by $f \rightarrow f(\omega), \omega \in \Omega$ and $f \in A$.

The relevant results from [1] will now be given. The norm on $B$ is given by

$$
\left\|\sum_{i=0}^{n-1} a_{i} x^{i}+(\alpha(x))\right\|=\sum_{i=0}^{n-1}\left|a_{i}\right| t^{i},
$$

Received by the editors October 23, 1961 and, in revised form, February 28, 1962.

1 The authors thank the referee for several useful suggestions. 
where $\alpha(x)=\sum_{i=0}^{n} \alpha_{i} x^{i}$ ( $\alpha_{n}=$ function identically one on $\Omega$ and)

$$
\sum_{i=0}^{n-1}\left|\alpha_{i}\right| t^{i} \leqq t^{n}
$$

(In the following, we assume without loss of generality that $t=1$.) The carrier space of $B$ is

$$
\Omega_{0}=\left\{(\omega, \lambda) \in \Omega \times C: \sum_{i=0}^{n} \alpha_{i}(\omega) \lambda^{i}=0\right\},
$$

endowed with the usual weak* topology, and the Gelfand representation $B^{\wedge}$ of $B$ is the algebra of functions $f$ continuous on $\Omega_{0}$ and which satisfy $f(\omega, \lambda)=\sum_{i=0}^{n-1} a_{i}(\omega) \lambda^{i}$, all $(\omega, \lambda)$ in $\Omega_{0}$, for some set $a_{0}, \cdots, a_{n-1}$ in $A$. (Note that if $(\omega, \lambda) \in \Omega_{0}$, then $|\lambda| \leqq 1$.)

In the case where $B$ is semi-simple, we may consider the norm $\|\cdot\|$ on $B$ as a norm on $B^{\wedge}$ so we will write $\|f\|=\sum_{i=0}^{n-1}\left|a_{i}\right|$, where the $a_{i}$ are the unique elements of $A$ such that $f(\omega, \lambda)=\sum_{i=0}^{n-1} \hat{a}_{i}(\omega) \lambda^{i},(\omega, \lambda)$ $\in \Omega_{0}$. Thus, under $\|\cdot\|, B^{\wedge}$ is a complete Banach algebra.

Let $\tau$ denote the continuous mapping of $\Omega_{0}$ onto $\Omega$ defined by $\tau(\omega, \lambda)=\omega,(\omega, \lambda) \in \Omega_{0}$. In [3], $\tau$ is shown to be an open mapping.

Theorem 1. Let $\Omega, \Omega_{0}, A$ and $\alpha(x)$ be as in the above. Suppose that $B$ is semi-simple. Then $B^{\wedge}$ is sup norm complete if and only if the discriminant $d$ of $\alpha(x)$ is not a topological divisor of zero in $A$ (i.e., there exists $s>0$ such that $|d a| \geqq s|a|$ for all $a$ in $A$ ).

Proof. Suppose that $d$ is not a topological divisor of zero in $A$. We will show that there exists a constant $K$ such that $\|f\| \leqq K|f|$, $f \in B^{\wedge}$ and from this it follows that $B^{\wedge}$ is complete under $|\cdot|$. To this end, for each $\omega \in \Omega$, let $\lambda_{1}(\omega), \cdots, \lambda_{n}(\omega)$ denote all the roots of $\sum_{i=0}^{n} \alpha_{i}(\omega) \lambda^{i}=0$, each root repeated according to its multiplicity. If

$$
f(\omega, \lambda)=\sum_{i=0}^{n-1} a_{i}(\omega) \lambda^{i} \quad(\omega, \lambda) \in \Omega_{0}, \quad \text { for each } \omega \in \Omega
$$

we view

$$
f\left(\omega, \lambda_{j}(\omega)\right)=\sum_{i=0}^{n-1} a_{i}(\omega) \lambda_{j}(\omega)^{i}, \quad j=1,2, \cdots, n
$$

as a system of $n$ linear equations in $a_{0}(\omega), \cdots, a_{n-1}(\omega)$. By Cramer's rule, we have that

$$
A(\omega) a_{i}(\omega)=\sum_{j=1}^{n} f\left(\omega, \lambda_{j}(\omega)\right) A_{j, i}(\omega), \quad i=0,1, \cdots, n-1
$$


where $A(\omega)$ is the van der Monde determinant of the $\lambda_{j}(\omega), j=1,2$, $\cdots, n$, and $A_{j, i}(\omega)$ is the cofactor of $\lambda_{j}(\omega)^{i}$ in that determinant. Since $A(\omega)^{2}=d(\omega)$, we have that

$$
d(\omega) a_{i}(\omega)=\sum_{j=1}^{n} f\left(\omega, \lambda_{j}(\omega)\right) A_{j, i}(\omega) A(\omega), \quad i=0,1, \cdots, n-1,
$$

so that $\left|d a_{i}\right| \leqq n(n !)^{2}|f|, i=0,1, \cdots, n-1$. Since $|d a| \geqq s|a|, a$ in $A$, it follows that

$$
|f| \leqq\|f\|=\sum_{i=0}^{n-1}\left|a_{i}\right| \leqq K|f|, \quad K=\left(\begin{array}{ll}
n & n !)^{2} / s .
\end{array}\right.
$$

Thus, $|\cdot|$ and $\|\cdot\|$ are equivalent norms on $B^{\wedge}$; hence, $B^{\wedge}$ is complete with respect to $|\cdot|$ since it is complete with respect to $\|\cdot\|$.

Suppose now that $B^{\wedge}$ is complete with respect to $|\cdot|$. Then $\|\cdot\|$ and $|\cdot|$ must be equivalent norms on $B^{\wedge}[4]$; i.e., there exists a constant $K$ such that $|f| \leqq\|f\| \leqq K|f|, f \in B^{\wedge}$. We will show that $d$ is not a topological divisor of zero in $A$ by assuming that it is and showing a contradiction. If $d$ is a topological divisor of zero in $A$, then there is a point $\omega_{0} \in \partial A$ (= Silov boundary of $\left.A ;[5]\right)$ such that $d\left(\omega_{0}\right)=0$. Let $\lambda_{1}, \cdots, \lambda_{m}(m<n)$ be the distinct roots of $\sum_{i=0}^{n} \alpha_{i}\left(\omega_{0}\right) \lambda^{i}$ $=0$ and define a function $y$ by

$$
y(\omega, \lambda)=\left(\lambda-\lambda_{1}\right)\left(\lambda-\lambda_{2}\right) \cdots\left(\lambda-\lambda_{m}\right), \quad(\omega, \lambda) \in \Omega_{0} .
$$

Clearly $y \in B^{\wedge}$. If $\epsilon$ is any positive number, then there exists a neighborhood $V_{\epsilon}$ in $\Omega$ of $\omega_{0}$ such that $(\omega, \lambda) \in \tau^{-1}\left(V_{\epsilon}\right)$ implies that $|y(\omega, \lambda)|$ $<\epsilon$. To see this, consider the function defined by $g(\omega)$ $=\sum_{i=1}^{n}\left|y\left(\omega, \lambda_{i}(\omega)\right)\right|, \omega \in \Omega$, and $\lambda_{i}(\omega)$ as above. Since $g$ is continuous and $g\left(\omega_{0}\right)=0$, there is a neighborhood $V_{\epsilon}$ of $\omega_{0}$ in $\Omega$ such that $g(\omega)<\epsilon$ if $\omega \in V_{\epsilon}$; hence, $|y(\omega, \lambda)|<\epsilon$ if $(\omega, \lambda) \in \tau^{-1}\left(V_{\epsilon}\right)$.

In [5], it is shown that there is a function $a_{\epsilon}$ in $A$ such that $\left|a_{\epsilon}\right|=1$ and $\left|a_{\epsilon}(\omega)\right|<\epsilon /|y|$ for $\omega \in V_{\epsilon}$. Thus, for all $(\omega, \lambda)$ in $\Omega_{0}$, we have that $\left|a_{\epsilon}(\omega) y(\omega, \lambda)\right|<\epsilon$; hence, $\left|a_{\epsilon} y\right|<\epsilon$. But $\left\|a_{\epsilon} y\right\| \geqq\left|a_{\epsilon}\right|=1$, so that $1 \leqq K\left|a_{\epsilon} y\right|<\epsilon K$, which is a contradiction. This completes the proof of the theorem.

3. Examples and further results. The condition that $B^{\wedge}$ be semisimple (i.e., $d$ not be a zero divisor) is not necessary for the completeness of $B^{\wedge}$. Witness the following example.

Example 1. Let $\Delta_{1}=\{\lambda \in C:|\lambda| \leqq 1\}, \Delta_{2}=\Delta_{1}+3$, and let $A=\left\{f \in C\left(\Delta_{1} \cup \Delta_{2}\right): f \mid \Delta_{1}\right.$ is analytic in the interior of $\Delta_{1}$ and $f(0)$ $=f(3)\}$. Then $\Delta_{1} \cup \Delta_{2}$ with the points 0 and 3 identified is the carrier space of $A$. If $f_{0}$ is the function defined by $f_{0}(\lambda)=\lambda$ if $\lambda \in \Delta_{1}$ and 
$f_{0}(\lambda)=0$ if $\lambda \in \Delta_{2}$, then $f_{0} \in A$. It is easily shown that $\left(A[x] /\left(x^{2}-f_{0}\right)\right)^{\wedge}$ is complete but $A[x] /\left(x^{2}-f_{0}\right)$ is not semi-simple since the discriminant $\left(=4 f_{0}\right)$ is a zero divisor in $A$.

Before we state the next result, let us define singular point of a polynomial over $A$. A point $\omega \in \Omega$ is said to be a singular point of $\alpha(x)$ if there exists a point $(\omega, \lambda) \in \Omega_{0}$ such that $\tau$ is not a local homeomorphism at $(\omega, \lambda)$. Now, each of the following statements is equivalent to the statement that $\tau$ is not a local homeomorphism at $(\omega, \lambda)$ :

(i) every neighborhood in $\Omega_{0}$ of $(\omega, \lambda)$ contains points $\left(\omega^{\prime}, \lambda^{\prime}\right)$ and ( $\left.\omega^{\prime}, \lambda^{\prime \prime}\right)$ such that $\lambda^{\prime} \neq \lambda^{\prime \prime}$ (since $\tau$ is an open mapping); or,

(ii) every neighborhood $V$ in $\Omega_{0}$ of $(\omega, \lambda)$ contains a point $\left(\omega^{\prime}, \lambda^{\prime}\right)$ such that the multiplicity of $\lambda^{\prime}$ as a root of $\sum_{i=0}^{n} \alpha_{i}\left(\omega^{\prime}\right) x^{i}=0$ is less than the multiplicity of $\lambda$ as a root of $\sum_{i=0}^{n} \alpha_{i}(\omega) x^{i}=0$ (see [3, Lemma $1.2])$.

Under the assumption that $\alpha(x)$ has no singular points, $\alpha(x)$ admits a factorization over $A$. Precisely, there exists idempotents $e_{1}, e_{2}, \cdots, e_{8}$ in $A$, polynomials $\alpha_{i j}$ in $A[x]$ and positive integers $k_{i j}, j=1,2, \cdots, m_{i}$; $i=1,2, \cdots, s$, such that

$$
\alpha(x)=\sum_{i=1}^{s} e_{i} \prod_{j=1}^{m_{i}} \alpha_{i j}(x)^{k_{i j}} .
$$

Furthermore, if $\beta_{i}(x)=e_{i} \prod_{j=1}^{m_{i}} \alpha_{i j}(x)$, then $\beta_{i}(x)$ is monic over, and has an invertible discriminant in, $e_{i} A$ (see [3] for details).

Theorem 2. Let $\Omega, \Omega_{0}, A$ and $\alpha(x)$ be as in the above. Suppose that $\alpha(x)$ has no singular points. Then $B^{\wedge}$ is complete.

Proof. It suffices to show that each $e_{i} B^{\wedge}$ is complete. But one easily shows that the algebra $e_{i} B^{\wedge}$ is isomorphic and isometric (sup norm) to the $\mathrm{Gel}^{\prime}$ fand representation of $B_{i}=\left(e_{i} A\right)[x] /\left(\beta_{i}(x)\right)$. Since $\beta_{i}(x)$ has an invertible discriminant in $e_{i} A$, it follows from Theorem 1 that $\hat{B_{i}}$ is sup norm complete. This proves the theorem. In the case where $B$ is semi-simple, Theorem 1 then asserts that $B^{\wedge}$ is complete if and only if no point of the Silov boundary is a singular point of $\alpha(x)$. Without the assumption that $B$ is semi-simple, this statement is false. First note that in Example $1, B^{\wedge}$ is complete, $B$ is not semi-simple and the point 0 in $\Delta_{1} \cup \Delta_{2}$ is the only singular point of $\alpha(x)$ and it is in the Silov boundary of $A$. To show the converse is false, we consider the case where $\alpha(x)=x^{2}-f_{0}, f_{0} \in A$, and $f_{0}$ not a zero divisor in $A$. In this case, this means that the null set of $f_{0}$ is open relative to the Silov boundary. Such a function $f_{0}$ is provided in:

EXAmple 2. Let $A_{0}$ denote the algebra of functions continuous on 
$\Delta=\{z \in C:|z| \leqq 1\}$ and analytic in the interior of $\Delta$. Let $A_{1}$ denote the algebra of functions which are uniform limits on $\Delta \times \Delta$ of polynomials in two complex variables and let $A_{2}=\left\{f \in C\left(\Omega_{1}\right): f \mid \Delta \times \Delta\right.$ is in $A_{1}$ and $\left.f \mid \Delta \in A_{0}\right\}$, where $\Omega_{1}=(\Delta \times \Delta) \cup \Delta$. Now, let $z_{n}=1 /(n+1)$ for $n \geqq 1$ and $z_{0}=0$, and $\zeta_{n}=\left(e^{z_{n}}, 0\right), n \geqq 0$. $A$ is to be the subalgebra of functions $f$ in $A_{2}$ such that $f\left(z_{n}\right)=f\left(\zeta_{n}\right)$ for all $n$. It is easily shown that each function in $A_{0}$ can be extended to a function in $A$. Because of the great freedom in extending such functions, it follows that the carrier space of $A$ is the space $\Omega_{1}$ with the points $z_{n}$ and $\zeta_{n}$ identified for each $n$ and that the Silov boundary of $A$ is the set

$$
\{z \in \Delta:|z|=1\} \cup\left\{\left(\eta_{1}, \eta_{2}\right) \in \Delta \times \Delta:\left|\eta_{i}\right|=1, i=1,2\right\} .
$$

For $f_{0}$ we take the function which is identically zero on $\Delta$ and equal to $\zeta_{2}$ for $\left(\zeta_{1}, \zeta_{2}\right) \in \Delta \times \Delta$. Then the null set of $f_{0}$ is open relative to $\partial A$ but $f_{0}$ is not a zero divisor in $A$, so that $B$ is semi-simple and $B^{\wedge}$ is not complete.

In the case where $A=C(\Omega)$, we can give a necessary and sufficient condition that $B^{\wedge}$ be the algebra $C\left(\Omega_{0}\right)$. In fact, we prove a more general result.

Theorem 3. Let $A=C(\Omega)$, and $\alpha(x)$ be a monic polynomial. If $K$ is a compact subset of $\Omega_{0}$, then $B^{\wedge} \mid K=C(K)$ if and only if $\tau \mid K$ is a local homeomorphism.

Proof. Suppose that $B^{\wedge} \mid K=C(K)$. We will show that $\tau \mid K$ is a local homeomorphism by showing that if it is not, then $B^{\wedge} \mid K \neq C(K)$. If $(\omega, \lambda) \in K$ is a point at which $\tau$ is not a local homeomorphism, then we can find points $\left(\omega_{n}, \lambda_{n}\right),\left(\omega_{n}, \mu_{n}\right), n>0$, in $K$ such that $\lambda_{n} \neq \mu_{n}$ and $\lambda_{n}, \mu_{n} \rightarrow \lambda$ as $n \rightarrow \infty$. Let $J$ denote this set of points. Then $J$ possesses at least one cluster point in $K$. If $\left(\omega^{\prime}, \lambda^{\prime}\right)$ is a cluster point of $J$, then $\lambda^{\prime}=\lambda$. We now define functions $\beta_{1}$ and $\beta_{2}$ on $\tau(\bar{J})$ as follows: $\beta_{1}\left(\omega^{\prime}\right)$ $=-\left(\lambda_{n}+\mu_{n}\right), \beta_{2}\left(\omega^{\prime}\right)=\lambda_{n} \mu_{n}$, if $\omega^{\prime}=\omega_{n}$, and $\beta_{1}\left(\omega^{\prime}\right)=-2 \lambda, \beta_{2}\left(\omega^{\prime}\right)=\lambda^{2}$, if $\left(\omega^{\prime}, \lambda\right)$ is a cluster point of $J$. Clearly, the $\beta_{i}$ are continuous on $\tau(\bar{J})$. By Theorem 1 (applied to the polynomial $\beta(x)=x^{2}+\beta_{1} x+\beta_{2}$ and the algebra $C(\tau(\bar{J}))$ we have that $B^{\wedge} \mid \bar{J}$ fails to be complete so that $B^{\wedge} \mid K$ cannot be complete since $\bar{J} \subseteq K$.

Now suppose that $\tau \mid K$ is a local homeomorphism. For each $(\omega, \lambda)$ $\in K$, there exists a compact neighborhood $V$ of $(\omega, \lambda)$ such that $\tau \mid V$ is a homeomorphism. Thus, $B^{\wedge} \mid V=C(V)$. Since $B^{\wedge}$ is a separating subalgebra of $C\left(\Omega_{0}\right), B^{\wedge} \mid K$ is a separating subalgebra of $C(K)$. Hence, if $x$ and $y$ are distinct points of $K$, there exists $f$ in $B^{\wedge}$ such that $f(x)$ $=1$ and $f(y)=0$. Thus, there exists a compact neighborhood $V$ in $K$ of $x$ such that $|f(z)|>\frac{1}{2}$ for $z \in V$, and $B^{\wedge} \mid V=C(V)$; hence, there 
exists a $g$ in $B^{\wedge} \mid K$ such that $f g \mid V=1$. It follows that $B^{\wedge} \mid K$ is a regular algebra (in the sense of Silov) and a standard argument (see $\left[4\right.$, proof of Lemma $25 \mathrm{E}$, p. 85]) implies that $B \mid K^{\wedge}=C(K) .^{2}$

Corollary. $B^{\wedge}=C\left(\Omega_{0}\right)$ if and only if $\alpha(x)$ has no singular points.

\section{REFERENCES}

1. R. Arens and K. Hoffman, Algebraic extensions of normed algebras, Proc. Amer. Math. Soc. 7 (1956), 203-210.

2. J. A. Lindberg, Jr., Algebraic extensions of commutative Banach algebras, (to appear).

3. - Polynomials over Banach algebras, (to appear).

4. L. H. Loomis, An introduction to abstract harmonic analysis, Van Nostrand, New York, 1953. 1960.

5. C. E. Rickart, General theory of Banach algebras, Van Nostrand, New York,

\section{UNIVERSITY OF NEBRASKA, \\ CONCORDia COLlEge AND YALE UNIVERSITY}

2 We are indebted to Professor I. Glicksberg for supplying us with the argument in this paragraph.

\section{THE MAXIMAL IDEAL SPACE OF THE FUNCTIONS LOCALLY IN A FUNCTION ALGEBRA}

\section{GABRIEL STOLZENBERG}

I. Introduction. If $A$ is a function algebra $^{1}$ with maximal ideal space, $M$, it is natural to consider those continuous functions on $M$ which belong "locally" to $A$. Precisely, if $f$ is a complex-valued continuous function on $M$, we shall say that $f$ is locally in $A$ provided that for each $m$ in $M$ there is a neighborhood, $U$ of $m$ and a function $a$ in $A$, such that $\left.f\right|_{U}=\left.a\right|_{U}$.

The outstanding problem concerning functions locally in $A$ is: Must every function which is locally in $A$ necessarily be a member of $A$ ? This note makes no significant contribution toward the solution of this problem. Its, very limited, aim is to study the maximal ideal space of the algebra generated by the functions which are locally in $A$. We prove, making essential use of the Local Maximum Modulus Principle [2], that this maximal ideal space is the same as the maximal

Received by the editors March 8, 1962.

1 Definitions are given in §II. 